\title{
The effect of tinea versicolor on thiol/disulphide homeostasis
}

\author{
Fadime Kilinc ${ }^{1}$, Ayse Akbas ${ }^{1}$, Sertac Sener ${ }^{1}$, Merve Ergin² $^{2}$ Pervin Baran ${ }^{3}$, Ahmet Metin $^{4}$ \\ 'Dermatology Clinic, Ankara Ataturk Training and Research Hospital, Ankara, Turkey \\ 2Department of Biochemistry, Gaziantep 25 Aralık State Hospital, Gaziantep, Turkey \\ ${ }^{3}$ Department of Biochemistry, Ankara Ataturk Training and Research Hospital, Ankara, Turkey \\ ${ }^{4}$ Dermatology Clinic, Ankara Yildirim Beyazit University, Medical Faculty, Ankara, Turkey
}

Adv Dermatol Allergol 2018; XXXV (3): 299-303

DOI: https://doi.org/10.5114/ada.2018.76227

\begin{abstract}
Introduction: Tinea versicolor is a superficial fungal infection caused by Malassezia spp. Malassezia spp. is a member of the normal human skin flora. It becomes a pathogen by transforming from the yeast form to the mycelium form. The oxidant/antioxidant homeostasis may be responsible for this. Thiol/disulphide homeostasis is a new marker indicating oxidative stress. This homeostasis is affected in many illnesses.

Aim: To investigate the thiol/disulphide homeostasis in patients with tinea versicolor.

Material and methods: Forty-two patients with tinea versicolor (median age: 36 years, min.-max.: 19-58) and 36 healthy controls (median age: 32 years, min.-max.: 18-60) were included in the trial. The levels of native thiol, disulphide, and total thiol were measured by an automated method in the patient and control groups. Disulphide/ total thiol, disulphide/native thiol and native thiol/total thiol rates were calculated as percentage.

Results: For the patient group and the control group, the native thiol levels were found to be $464.32 \pm 51.48 \mathrm{mmol} / \mathrm{l}$ and $465.18 \pm 51.32 \mathrm{mmol} / \mathrm{l}$, disulphide levels $-19.80 \pm 7.08 \mathrm{mmol} / \mathrm{l}$ and $21.27 \pm 8.90 \mathrm{mmol} / \mathrm{l}$, total thiol levels -503.92 $\pm 53.65 \mathrm{mmol} / \mathrm{l}$ and $508.07 \pm 56.59 \mathrm{mmol} / \mathrm{l}$, respectively. No statistical difference was detected between the two groups.

Conclusions: Thiol/disulphide homeostasis was not affected in tinea versicolor. According to our findings, oxidative stress seems to have no role in the pathogenesis of tinea versicolor.
\end{abstract}

Key words: oxidative stress, thiol disulphide homeostasis, tinea versicolor.

\section{Introduction}

Tinea versicolor (TV) is a superficial fungal infection caused by Malassezia spp. and is characterized by hypopigmented or hyperpigmented macules localized on the face, arms or body with a clinical manifestation of associated mild desquamation [1-3]. Macules are generally of 3-5 $\mathrm{mm}$ in diameter, round or oval, may affect large areas and be displayed in irregular forms [1]. It is more common in tropical climates. While it frequently affects young adults, it can be seen at every age, even in new-borns. It is asymptomatic, and may rarely cause pruritus [3].

Free radicals form disulphide bonds by oxidizing the thiol groups in proteins containing sulphur. Being the earliest sign of protein oxidation, these bonds are reduced to thiol groups again when necessary, and this ensures the thiol/disulphide homeostasis [4]. This homeostasis is affected in many illnesses [4-11] and it has never been researched before in patients with TV.

Malassezia spp. is a member of the normal human skin flora. They are dimorphic fungi which can exist both as yeast or mycelium. It has 14 lipophilic types [12-15]. It has a multi-layered thick cell wall containing lipid. The lipid content may be removed using solvents. It requires fatty acids to grow $[16,17]$. It exists in the yeast form on normal skin $[12,13]$. It becomes a pathogen by transforming from the yeast form to the mycelium form [2]. However, the mechanism through which this transformation occurs and these spp. exert an effect on the skin still remains unknown.

Address for correspondence: Fadime Kilinc MD, Dermatology Clinic, Ankara Ataturk Training and Research Hospital, Yasamkent mah. 3254. cd. Crystal Towers Sitesi D4, 1606810 Cayyolu, Ankara, Turkey, phone: +90 (312) 2171709, +90 05056842806 , fax: +90 (312) 2912705, e-mail:fykilinc@yahoo.com Received: 3.07.2017, accepted: 25.07.2017. 


\section{Aim}

In our study, we hypothesized that the oxidant/antioxidant homeostasis of the organism may be responsible for this, and investigated thiol/disulphide homeostasis in patients with TV.

\section{Material and methods}

The trial was performed in accordance with good clinical practice and Helsinki Declaration. The patients and the controls participating in the study were informed about the trial and provided consent forms. Approval was granted by the local ethics committee of the Yildirim Beyazit University. The study included 42 patients over 18 years old who were admitted to our outpatient clinic and clinically diagnosed with TV with a positive result in native preparation prepared with $10 \%$ potassium hydroxide in microscopy, and did not use medication within the last three months, and 36 healthy subjects as the control group. The patients who are pregnant, or have the history of systemic disease, medication use or smoking

Table 1. The demographic characteristics of the patient and control groups

\begin{tabular}{lccc}
\hline $\begin{array}{l}\text { Demographic } \\
\text { characteristics }\end{array}$ & $\begin{array}{c}\text { Patients } \\
(n=42)\end{array}$ & $\begin{array}{c}\text { Controls } \\
(n=36)\end{array}$ & P-value \\
\hline $\begin{array}{l}\text { Age, mean } \pm \text { standard } \\
\text { deviation }\end{array}$ & $35.88 \pm 10.29$ & $34.75 \pm 10.60$ & 0.637 \\
\hline $\begin{array}{l}\text { Median (min.-max.) } \\
\text { Gender, } n \text { (\%): }\end{array}$ & $36(19-58)$ & $32(18-60)$ & \\
\hline \begin{tabular}{l} 
Male \\
\hline Female
\end{tabular} & $22(52.4)$ & $18(50.0)$ & 1.000 \\
\hline $\begin{array}{l}\text { Disease duration [month] } \\
\text { Median (min.-max.) }\end{array}$ & $12(1-168)$ & - & - \\
\hline $\begin{array}{l}\text { Family history } \\
\text { Recurrence }\end{array}$ & $6(14.3)$ & - & - \\
\hline \begin{tabular}{l} 
Shoulder \\
\hline Nape
\end{tabular} & $23(54.8)$ & - & - \\
\hline $\begin{array}{l}\text { Buritus } \\
\text { Color: }\end{array}$ & $13(31.0)$ & - & - \\
\hline Hyperpigmentation & $31(73.8)$ & - & - \\
\hline Hypopigmentation & $11(26.2)$ & - & - \\
\hline
\end{tabular}

were excluded. Information regarding the age, sex, duration of the disease, the number and localization of the lesions, pigmentation status, recurrence and family history of the patients were recorded. Lesions were graded in 3 groups according to their distribution on body parts. If the lesions were localized in one body part, they were considered to be mild, if localized in 3 or less body parts, they were considered to be moderate, and if they were in more than 3 body parts they were considered to be disseminated.

Venous blood samples were collected into biochemistry tubes after overnight fasting. They were centrifuged at $3500 \mathrm{rpm}$ for $10 \mathrm{~min}$, and stored in deep freezer (Sanyo, Japan) at $-80^{\circ} \mathrm{C}$.

Thiol/disulphide homeostasis was assessed using an automated spectrometric method recently developed by Erel and Neselioglu [11]. In this method, firstly, disulphide bonds were reduced to free thiol groups using $\mathrm{NaBH} 4$. Unused $\mathrm{NaBH} 4$ was treated with formaldehyde, thereby, reduction of 5,5'-dithiobis-(2 nitrobenzoic) (DTNB) was prevented. After the reaction with DTNB, total thiol groups (the sum of native and reduced thiol) were calculated, and the amount of disulphide was calculated by dividing the difference between total thiol and native thiol by two. Disulphide/total thiol, disulphide/native thiol and native thiol/total thiol rates were calculated as percentages. Cobas c501 analyser (Roche Diagnostics, Mannheim, Germany) was used for the measurement. Thiol and disulphide values were expressed as $\mathrm{mmol} / \mathrm{l}$.

\section{Statistical analysis}

The distribution of the age, native thiol, disulphide, total thiol, disulphide/total thiol, disulphide/native thiol and native thiol/total thiol variables in the study was explored visually and using Shapiro-Wilk test. As these variables are non-normally distributed, they were expressed as mean \pm standard deviation (mean \pm SD) and median (minimum-maximum). Sex, localization, recurrence, family history, pigmentation and presence of pruritus were expressed in numbers (\%).

The patient group and the control group were compared in terms of age, native thiol, disulphide, total thiol, disulphide/total thiol, disulphide/native thiol and native thiol/total thiol, and these parameters were compared in the patient group by Mann-Whitney $U$ test using sex, family history, pigmentation, recurrence and pruritus as factors. The sex distribution of the groups was evaluated by $\chi^{2}$ test. The relationship between age and disease duration, and native thiol, disulphide and total thiol were explored by Spearman's correlation analysis. The statistical significance level was accepted to be $p<0.05$.

IBM SPSS Statistics 21.0 (IBM Corp. Released 2012. IBM SPSS Statistics for Windows, Version 21.0. Armonk, NY: IBM Corp.) software was used for statistical analyses and calculations. 
Table 2. Thiol and disulphide levels in patients and controls

\begin{tabular}{lccc}
\hline Parameter & Patients $(n=42)$ & Controls $(n=36)$ & $P$-value \\
\hline Native thiol, mean \pm SD $[\mathrm{mmol} / \mathrm{l}]$ & $464.32 \pm 51.48$ & $465.18 \pm 51.32$ & 0.814 \\
\hline Median (min.-max.) & $468.25(361.60-586.50)$ & $463.70(319.70-551.10)$ & \\
\hline Disulphide, mean \pm SD [mmol/l] & $19.80 \pm 7.08$ & $21.27 \pm 8.90$ & 0.336 \\
\hline Median (min.-max.) & $20.50(1.20-32.35)$ & $22.15(1.05-35.25)$ & 0.528 \\
\hline Total thiol, mean \pm SD [mmol/l] & $503.92 \pm 53.65$ & $508.07 \pm 56.59$ & 0.362 \\
\hline Median (min.-max.) & $509.65(397.50-617.20)$ & $516.65(349.70-611.20)$ & 0.362 \\
\hline D/T, mean \pm SD (\%) & $0.039 \pm 0.014$ & $0.042 \pm 0.016$ & \\
\hline Median (min.-max.) & $0.040(0.002-0.070)$ & $0.044(0.002-0.069)$ & 0.378 \\
\hline D/N, mean \pm SD (\%) & $0.043 \pm 0.016$ & $0.046 \pm 0.019$ & \\
\hline Median (min.-max.) & $0.044(0.002-0.082)$ & $0.048(0.002-0.082)$ & \\
\hline N/T, mean \pm SD (\%) & $0.921 \pm 0.028$ & $0.916 \pm 0.034$ & \\
\hline Median (min.-max.) & $0.920(0.859-0.995)$ & $0.914(0.847-0.996)$ & \\
\hline
\end{tabular}

$D / T$ - disulphide/total thiol, D/N - disulphide/native thiol, N/T - native thiol/total thiol.

\section{Results}

The median age of the patients and the controls was calculated to be 36 years (min.-max.: 19-58) and 32 years (min.-max.: 18-60), respectively (Table 1). $52.4 \%(n=22)$ of the patient group, and $50.0 \%(n=18)$ of the control group were males. The age and sex distribution of the patient and control groups was comparable $(p>0.05)$. Information on and clinical characteristics of the patients are shown in Table 1; and 30 patients were in mild, 11 in moderate and only one patient in the disseminated category.

Native thiol medians of the patient and control groups were calculated to be $468.25 \mathrm{mmol} / \mathrm{l}$ (min.-max.: 361.60-586.50) and $463.70 \mathrm{mmol} / \mathrm{l}$ (min.-max.: 319.70551.10), respectively (Table 2 ). No significant difference was found between the two groups in terms of native thiol $(p=0.814)$. Two groups were also comparable in terms of other parameters $(p>0.05)$.

When thiol, disulphide and total thiol assessments in the patient group were compared for sex, family history, recurrence, pigmentation and pruritus status and grading, no statistically significant difference was found ( $p>0.05$ ).

When the relationships between thiol measurements, and age and disease duration were evaluated, a moderately significant negative relationship was found between age, and native thiol and total thiol (Spearman rho: $-0.415, p=0.006)$. No statistically significant relationship was found between age, and other thiol measurements and disease duration $(p>0.05)$.

\section{Discussion}

In this study, dynamic thiol/disulphide homeostasis, which is the oxidative stress marker, was investigated for the first time in patients with TV. Thiol/disulphide homeostasis was measured by a method which has been developed by Erel and Neselioglu and gives rapid and accurate results. When we compared the native thiol, disulphide and total thiol values, and disulphide/total thiol, disulphide/native thiol and native thiol/total thiol rates of the patients with TV and the control group, we could not detect a significant difference and thought that thiol/ disulphide homeostasis is maintained in these patients.

The Malassezia spp. causing superficial mycosis are colonized in seborrheic regions in humans. As they are lipophilic, the presence of fatty acids on the skin helps them grow, and the increase in skin lipids due to sex hormones in adolescents leads to increased Malassezia spp. Being a part of the normal skin flora, Malassezia spp. may cause cutaneous and systemic diseases due to environmental modifications and individual immunological changes [1]. In addition to TV, seborrheic dermatitis and Malassezia folliculitis, they are also believed to be responsible for the pathogenesis of skin diseases such as atopic dermatitis and psoriasis [12].

Normally, Malassezia spp. have commensal balance in the organism, upon the impairment of this balance, the yeast forms become pathogenic. The reason behind this could not be explained completely. It has been suggested that the lack of inflammation when the fungi are at commensal form may be due to cell wall lipids, and cellular immune response is also important. In a study, it has been stated to suppress the cytokine production from mononuclear cells, and the azelaic acid produced by it decrease the reactive oxygen intermediate products [16]. When the lipid layer is removed, the cytokine suppression has been observed to disappear, and Malassezia 
(M) globosa and M. slooffiae spp. have been observed to increase the secretion of IL-1 $\alpha, M$. furfur, M. obtusa, $M$. restricta and $M$. sympoidalis spp. have been observed to increase the secretion of IL-6, and all spp. have been observed to increase the secretion of IL-8 [17].

Clinically, the most frequently isolated Malassezia spp. are M. globosa and M. furfur [1]. Tinea versicolor is believed to develop on a genetic basis [18]. It is believed that the factors implicated for the development of TV may differ for each individual, and Malassezia spp. may switch from the saprophytic phase to the pathogenic phase due to factors including high temperature, humidity, oily skin, sweating, heredity and immunosuppression $[1,12]$. Putting oily creams and corticosteroids on the body, exposure to sunlight and malnutrition are the other factors implicated in this process [1].

Malassezia spp. have been suggested to be able to activate the complement system via the classical or the alternative pathways, which is responsible for the inflammation [16]. However, despite a high increase in Malassezia spp. in TV, inflammation does not occur [19]. And the indole compounds produced by them are responsible for this [17]. Epidermal barrier dysfunction is present in all the skin diseases except folliculitis [18]. The expression of the antimicrobial peptide, katelicidin (LL37) which is important for the host response against fungi in keratinocytes is increased in TV [17].

In the histopathological examinations of the TV lesions, Malassezia yeasts were observed in all layers of stratum corneum and the presence of many Langerhans cells was observed in the epidermis. Free radicals are released during the phagocytosis of the microorganisms by macrophages. Furthermore, macrophages inhibit the growth of fungal pathogens by producing nitric oxide. Neutrophils and mononuclear cells are important for the defence against Malassezia. The phagocytosis of Malassezia by neutrophils is only $5 \%$. And this is thought to be caused by the lipid layer on the cell wall [16].

There is no other study evaluating the thiol/disulphide homeostasis in patients with TV. However, there are two studies on the role of the oxidative stress in the pathogenesis $[2,19]$. Kurutas and Ozturk investigated the oxidative and nitrosative stress in the lesions of patients with TV, and found the levels of malondialdehyde, nitric oxide and nitrotyrosine, thereby, the oxidative and nitrosative stress increased, and observed an increase in the activities of the antioxidant defence enzymes, superoxide dismutase and catalase in response to this [2]. Kurutas and Ozturk observed the presence of local oxidative stress in patients with TV via lipid peroxidation, and we aimed to evaluate the oxidative stress in patients with $T V$ via protein oxidation. However, it was found unaffected. This suggests that lipids have more important roles in pathogenesis.

In their immunohistochemical examination of the lesions of patients with TV, Kilic et al. found that glu- tathione s-transferase isoenzyme M4 is decreased and glutathione s-transferase isoenzyme $\mathrm{T} 1$ is increased, and thought that oxidative stress may be effective in the pathogenesis [19].

In these two studies, local oxidative stress was investigated in patients with TV and found to be increased, we wanted to measure the total oxidative state systemically and found that it was not affected.

\section{Conclusions}

In our study, we evaluated the thiol/disulphide homeostasis, which is recognized as a new marker for oxidative stress, by measuring the levels of thiol and disulphide in patients' serum, and found that homeostasis is unaffected. And, this indicates that there may be different factors other than the oxidative stress for Malassezia spp. to gain pathogenicity, therefore, further research is needed. Furthermore, new studies are needed together with local and systemic oxidative stress that has been investigated.

\section{Conflict of interest}

The authors declare no conflict of interest.

\section{References}

1. Mendez-Tovar LJ. Pathogenesis of dermatophytosis and tinea versicolor. Clin Dermatol 2010; 28: 185-9.

2. Kurutas EB, Ozturk P. The evaluation of local oxidative/nitrosative stress in patients with pityriasis versicolor: a preliminary study. Mycoses 2016; 59: 720-5.

3. Bonifaz A, Gómez-Daza F, Paredes V, Ponce RM. Tinea versicolor, tinea nigra, white piedra, and black piedra. Clin Dermatol 2010; 28: 140-5.

4. Ates I, Altay M, Yilmaz FM, et al. Dynamic thiol/disulfide homeostasis in patients with autoimmune subclinical hypothyroidism. Endocr Res 2016; 41: 343-9.

5. Topuz M, Şen O, Kaplan M, et al. The role of thiol/disulphide homeostasis in anthracycline associated cardiac toxicity. Int Heart J 2017; 58: 69-72.

6. Dogru A, Balkarli A, Cetin GY, et al. Thiol/disulfide homeostasis in patients with ankylosing spondylitis. Bosn J Basic Med Sci 2016; 16: 187-92.

7. Gumusyayla S, Vural G, Bektas H, et al. A novel oxidative stress marker in migraine patients: dynamic thiol-disulphidehomeostasis. Neurol Sci 2016; 37: 1311-7.

8. Ergin M, Cendek BD, Neselioglu S, et al. Dynamic thiol-disulfide homeostasis in hyperemesis gravidarum. J Perinatol 2015; 35: 788-92.

9. Akbas A, Kilinc F, Sener S, et al. Investigation of thiol-disulphide balance in patients with acute urticaria and chronic spontaneous urticaria. Cutan Ocul Toxicol 2017; 36: 205-10.

10. Demirseren DD, Cicek C, Alisik M, et al. Dynamic thiol/disulphide homeostasis in patients with basal cell carcinoma. Cutan Ocul Toxicol 2017; 36: 278-82.

11. Erel O, Neselioglu S. A novel and automated assay for thiol/ disulphide homeostasis. Clin Biochem 2014; 47: 326-32. 
12. Ljubojević S, Skerlev M, Lipozencić J, Basta-Juzbasić A. The role of Malassezia furfur in dermatology. Clin Dermatol 2002; 20: 179-82.

13. Emre S, Metin A, Demirseren DD, et al. The association of oxidative stress and disease activity in seborrheic dermatitis. Arch Dermatol Res 2012; 304: 683-7.

14. Prohić A, Jovović Sadiković T, Kuskunović-Vlahovljak S, Baljić R. Distribution of Malassezia species in patients with different dermatological disorders and healthy individuals. Acta Dermatovenerol Croat 2016; 24: 274-81.

15. Gaitanis G, Magiatis P, Hantschke M, et al. The Malassezia genus in skin and systemic diseases. Clin Microbiol Rev 2012; 25: 106-41.

16. Kantarcıoğlu SA, Yücel A. Malassezia species: taxonomy, mycology, immunology, pathogenesis, distrubution and related infections, laboratory diagnosis, antifungal susceptibility. Cerrahpaşa J Med 2005; 36: 134-54.

17. Ashbee HR. Update on the genus Malassezia. Med Mycol 2007; 45: 287-303.

18. Gaitanis G, Velegraki A, Mayser P, Bassukas ID. Skin diseases associated with Malassezia yeasts: facts and controversies. Clin Dermatol 2013; 31: 455-63.

19. Kilic M, Oguztuzun S, Karadag AS, et al. Expression of GSTM4 and GSTT1 in patients with Tinea versicolor, Tinea inguinalis and Tinea pedis infections: a preliminary study. Clin Exp Dermatol 2011; 36: 590-4. 